\title{
Editorial: John Ozimek, newly appointed Editor of the Journal of Database Marketing, sets out his plans for the Journal's future
}

There is nothing like taking over in mid-stream: not just changing the tanker's course, but parachuting onto the bridge two days after it has left port. For a change of course is coming. While we cannot guarantee to satisfy all of the readers all of the time, we believe it will be broadly welcome, as it will bring the Journal of Database Marketing more in tune with the 21 st century.

For myself, this is not entirely new territory. I have been closely associated with the Journal since its inception. I have watched it grow from an idea about database marketing, becoming, over time, what it is today: the main, perhaps only, serious European journal on the subject of database marketing and, by extension, on relationship marketing. In that, thanks go out to each and every one of the previous editorial team.

The Journal is read by thousands and photocopied by many more - some of whom even pay for that privilege! We have a solid subscriber base and a talented and enthusiastic editorial board. Yet we are still, in some sense, rooted in our past. Databases and database technology are important, but they are also a part of the whole that is now customer management strategy (CMS). Over the last few years, the Journal's scope has expanded constantly, but surreptitiously. Without acknowledging it overtly, the Journal has been shifting its focus towards CMS and the underpinning strategies that are essential to that task, but it has not owned up to that shift. That is an issue, because dissonance between claim and reality always will be found out eventually. Therefore, we are well past the time when we should acknowledge that the Journal, in future, will be focusing more explicitly on issues of CMS. Some of the topics we plan to cover are:

— risk management (and integration with credit risk planning)

- next best action

- multi-channel

- customer value

- research-based CRM

- customer decisioning models

- segmentation (competitor strategy)

- culture change

- programme management

- e-data.

This is far from an exhaustive list. It will change as the emphasis within CM changes. Undoubtedly, you will have your own views on what else should be in there - and what you would leave out. If you do, please write and let us know. Even better, write and then talk to us about producing a paper on the subject.

We will retain and strengthen our dedication to academic rigour. At the 
same time, we will be redoubling efforts to bring you experience of what has worked. Two questions will be asked of all future material:

- does this advance our thinking in the field

- what difference did this approach make to the company that attempted it and what lessons are to be learned?

Less traditional approaches will be tested. The paper in this issue from Jim Laiderman is not quite a model of academic style. However, it is an expert view produced by an expert practitioner, communicating his real and practical experience of an approach to business segmentation that has worked. As such, we believe it belongs in the Journal.

We will be doing a great deal more to stimulate debate and interaction in future issues. We will also be looking at particular themes:

- media neutral planning

- data mining

- synergy between business and academia and how they can help each other.

To return to the present issue. The paper from Moskowitz et al. looks at the messages conveyed by six different automobile models in order to identify the type of strategy adopted by the manufacturer of each model. One significant aspect of this paper is that the approach utilises a technique known as 'conjoint analysis' - a favourite of market researchers, but not so often seen in the direct and database fields. It serves to help organisations understand the trade-off between a variety of benefits.

Shena Mitchell tackles the old problem of unsolicited mail from a new angle: attempting to create and understand segments that are most sensitive to the issue. Xueming Luo looks at how the provision of customised and contextual information to customers at the point of need in real time can become the key to reaching and retaining online customers. Merlin Stone et al. continue their excellent exploration of the theory and concepts of data quality management by applying research into how data are actually used and their impact on companies that use them. Finally, Debra Zahay and Abbie Griffin have reviewed the areas of customisation and personalisation to come up with some constructs that underpin success in this area. One intriguing finding, perhaps buried within the paper, is that companies with a long tradition of direct marketing may well be better at CM than those which lack that tradition. Obvious, perhaps, but not always sufficiently acknowledged by strategists who think that one can graft CM onto an above-the-line management as easily as a new brand campaign.

We always welcome feedback on any and every aspect of the Journal. As this editorial suggests, change is now in the air - not least with regard to the Journal's title. So if you have any thoughts on the subject, please let us know in writing or by e-mail to the editor directly at emcity@easynet.co.uk.

JOHN OZIMEK Editor

February 2003 